\title{
The cortical connection
}

\author{
BENJAMIN SCHOLL and NICHOLAS J. PRIEBE \\ Department of Neuroscience, The University of Texas, Austin, Texas 78712, USA.
}

Social-networking websites such as Facebook allow us to connect with people all over the world, but as our list of friends grows, it becomes difficult to read and react to posts from everyone. Instead, we often choose to respond primarily to those people with whom we share common interests, perhaps close family and friends. Similarly, neurons in the brain's cerebral cortex receive between 1,000 and 10,000 synaptic connections, from hundreds or thousands of other neurons ${ }^{1}$. But it is not known whether neurons listen to all of those inputs equally. Could certain inputs be more influential than others? In this issue, Cossell et al. ${ }^{2}$ (page 399) demonstrate that neurons in the visual cortex listen to a subset of their synaptic inputs, and that the neurons comprising that subset have strikingly similar functional properties to the target neuron - much like how our closest Facebook friends have similar interests to our own.

For decades, neuroscientists have attempted to uncover the relationship between a neuron's functional properties and its synaptic input from other neurons. On the one hand, studying this relationship in vivo is challenging, because although it is possible to determine which sensory stimuli excite each neuron (known as its receptive field), it is difficult to identify synaptic inputs. On the other hand, the gold standard for measuring synaptic inputs is to make direct measurements in vitro using simultaneous recordings from pairs of neurons in brain slices, where it is not possible to measure the receptive field. Cossell and colleagues confront this challenge by combining measurements taken in vivo and in vitro to reveal the receptive fields of synaptically connected neurons. The authors discover that neurons receive inputs from a diverse population of neighbours, but that most inputs are drowned out by a few dominant synapses.

Given the enormous number of synaptic connections identified from anatomical measurements, it is puzzling that only a few synaptic inputs dominate. Cossell and coworkers uncover a direct relationship between connection strength (known as a synaptic weight) and receptive field in connected pairs of neurons. The few strong synaptic connections originate from neurons with receptive fields matching the neuron onto which they synapse. By contrast, many — but weak — synaptic connections originate from presynaptic neurons with different receptive fields (Fig. 1).

If only a few synaptic inputs drive neuronal responses, why is there such dense connectivity in the cerebral cortex? Perhaps this wiring is useful for brain development and plasticity (changes in neuronal connections), or even for the formation of memories. The presence of 
many weak connections might allow cortical networks to be reprogrammed easily. For instance, modulating the weighting of just a few key synaptic inputs could induce dramatic changes in a neuron's receptive field.

Changing the receptive field of cortical neurons by generating new anatomical connections would take time and resources. By having those connections in place, and to some degree dormant, neuronal plasticity can be achieved quickly. Furthermore, because the inputs are already in place, changes in synaptic weight during associative learning can be driven by mechanisms such as Hebbian plasticity, in which correlated activity of a presynaptic neuron with that of the postsynaptic neuron increases the synaptic weight ${ }^{3,4}$. An excess of synaptic contacts might therefore be key to plasticity, which occurs in mature cortical circuits ${ }^{5,6}$.

Both the potential plasticity of cortical circuits and the non-uniform nature of synaptic input strengths present challenges to connectome projects, which aim to determine the functional properties of neurons on the basis of anatomical connectivity alone ${ }^{7-9}$. Consider an attempt to decode the personality traits of a single person on the basis of their full set of Facebook friends. This would be a tough job, given the wide variety of individuals in the group, which might include friends, family and acquaintances. Similarly, if we assume that all connections are equivalent in a neuronal network, predicting the response selectivity of a cortical neuron may be difficult, because the inputs are so diverse. Although connectome projects will certainly generate valuable statistics about connectivity in the cerebral cortex, Cossell and colleagues demonstrate that identifying the weights of synaptic connections is essential to account for neuronal responses. When connectivity is considered in conjunction with synaptic weighting, it should be possible to predict response selectivity.

Despite the difficulties associated with determining connection weight from anatomical measurements alone, there are hints that not all synaptic inputs are physically equal. Properties of synaptic contacts that may be linked to synaptic weight ${ }^{10}$ include the size of postsynaptic structures such as dendritic spines, the number of neurotransmitter molecules available for release by the presynaptic terminal, and the extent to which subcellular compartments that are responsible for protein synthesis can form new postsynaptic structures. Understanding these links will be crucial for bridging the gap between functional and anatomical connectivity, so that neuroscientists can get closer to obtaining a functional connectome.

\section{References}

1. Binzegger T, Douglas RJ \& Martin KA C. J. Neurosci 24, 8441-8453 (2004).

2. Cossell L et al. Nature 518, 399-403 (2015). [PubMed: 25652823]

3. Isaac JTR, Nicoll RA \& Malenka RC Neuron 15, 427-434 (1995). [PubMed: 7646894]

4. Liao D, Hessler NA \& Malinow R Nature 375, 400-404 (1995). [PubMed: 7760933]

5. Allard T, Clark SA, Jenkins WM \& Merzenich MM J. Neurophysiol 66, 1048-1058 (1991). [PubMed: 1753275]

6. Sato M \& Stryker MP J. Neurosci 28, 10278-10286 (2008). [PubMed: 18842887]

7. Kleinfeld D et al. J. Neurosci 31, 16125-16138 (2011). [PubMed: 22072665]

8. Lichtman JW, Livet J \& Sanes JR Nature Rev. Neurosci 9, 417-422 (2008). [PubMed: 18446160]

9. Oh SW et al. Nature 508, 207-214 (2014). [PubMed: 24695228] 
10. Bourne JN \& Harris KM Annu. Rev. Neurosci 31, 47-67 (2008). [PubMed: 18284372]

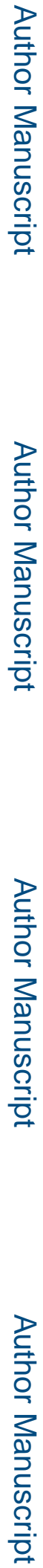

Nature. Author manuscript; available in PMC 2018 December 30. 

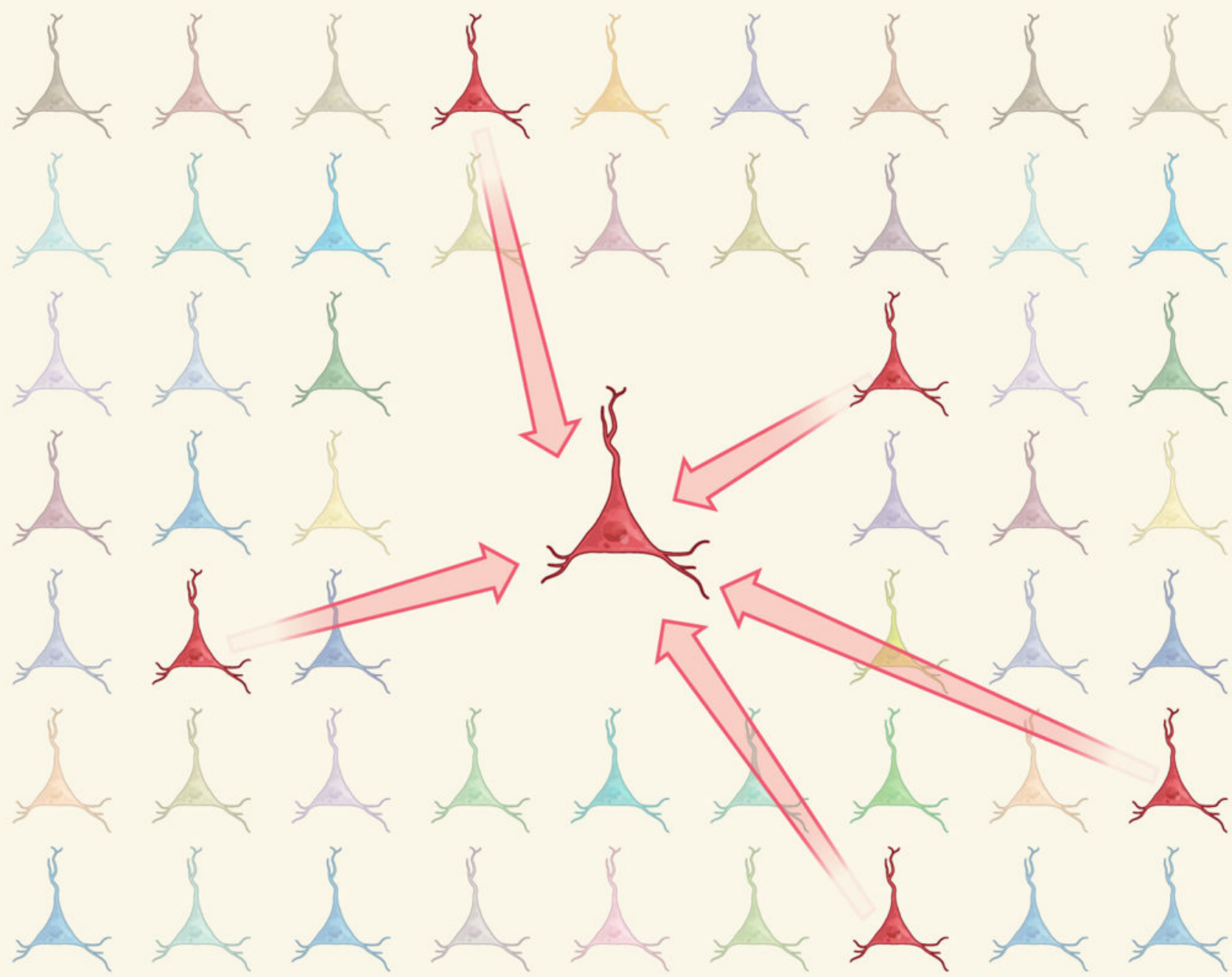

Figure 1 |. Deciphering neuronal networks.

Neurons in the visual cortex receive synaptic inputs from many other neurons, here represented in a grid. Neurons activated by similar sensory stimuli - that is, those with similar receptive fields - are depicted in the same colour. The large target neuron in the centre receives connections from all the other neurons in the grid (connections not shown). If all of those connections are weighted equally, it is difficult to determine the function of the target neuron. But Cossell et al. ${ }^{2}$ report that the target neuron receives strong inputs (indicated by arrows) only from neurons with similar receptive fields to its own. 